\title{
Research of Distribution Network Expansion Planning With Wind Power
}

\author{
Jiheng Jiang \\ North China Electric Power University, Beijing, China \\ savoryly@163.com \\ 2nd Beinong Road, Huilongguan, Changping District, Beijing
}

Keywords: wind power; distribution network; system with wind-light

Abstract. The paper analyses the distribution network wind power access two modes: wind power accessory construction energy storage equipment, mainly in the wind power, and photo voltaic generation, supplemented by the scenery of the joint system reservoir. According to two modes respectively established the expected value model to include line investment cost of operation, the wind power system investment cost of operation, power purchase cost and environmental costs, of the total social cost minimization, according to the system as the objective function of the load growth situation, solve the distribution network expansion planning more wind power system, line, and the location of the combinatorial optimization problem of let.

\section{Introduction}

With the increasingly serious environmental pollution and energy crisis, renewable energy gradually occupies an important position in the national energy strategy. In addition to hydropower, wind power is the most mature technology development, development cost minimum, the future is the most value of large-scale development of renewable energy. At present, most of the wind turbine directly run in the distribution network, making the distribution network planning is no longer a traditional power system planning, but closely related to the national sustainable development strategy and energy policy of resource optimal allocation. Wind power access, can reduce the network loss and delay power grid upgrading and save conventional energy consumption, reduce pollution emissions, but by the impact of weather and other natural conditions, its output has obvious randomness, the traditional planning method is no longer applicable.

\section{Wind Power Distribution Network Model}

With the continuous expansion of the scale of wind power into the grid, wind power characteristics on the stability of the system [1] and electric energy quality [2-3] brings negative effect will be more significant. There are 2 ways to solve:

(1)Constructing the reasonable capacity and to form a complete set of energy storage devices, and thus on the one hand, make the wind as the output power for a long time stability, on the other hand can play a positive role to scheduling and operation of power grids. However, the current development of larger and only pumped storage energy storage technology, other technical development level is low, can't meet the demand of power energy storage reality effectively.

(2) The introduction of wind power has the time complementary photovoltaic, and configure the appropriate storage capacity than a light, effective control of comprehensive cost.

\section{Wind-Storage Mode of Distribution Network Planning}

Energy storage equipment capacity Section headings. Installation of energy storage device to a certain extent, improve output has ever will have obvious fluctuation problem, but to determine a reasonable storage capacity, is too small effect on the improvement of the fluctuation is not obvious; general principle against economy; In addition, pay attention to the rationality of the energy storage equipment capacity of wind power the effective use and cost effective control requirements. Through 
real-time energy storage compensation method [4] to determine the energy storage equipment capacity formula, and the schematics of the in the optimal selection of power distribution network planning.

(1) Using Monte Carlo method to get wind of annual output expectations, and its output power setting for the wind storage system plan.

(2) On the basis of the above, identify the output value corresponding to the number of wind speed Rated wind speed value where $v_{0}$, the value must be a fan is smaller.

(3) In the wind-system operation in the process of storage, generally will be to where $v_{0}$ as fan start wind speed values, if the wind speed is greater than the value of the system system in accordance with the corresponding power value where $v_{0}$ output can, at the same time will beyond the numerical part of the energy from the wind farm in the storage to the energy storage system, if wind speed is less than where $v_{0}$, lack of energy provided by the energy storage system, the whole system is still under wind speed where $\mathrm{v}_{0}$ corresponding power output value.

(4) The capacity of the energy storage device can be calculated using the type:

$$
S^{\text {rated }}=P_{w} H
$$

In the type: the $P_{w}$ is the expected output value for wind farm 1 year; $H$ for the wind farm as the number of hours. When wind speed for a long time the start wind speed is low, the Parameters to determine whether the output power of the energy storage system stability.

Distribution network planning model. Objective function mainly includes the following content: (1) The line additional annual fee use and its running cost, in this use $C_{L}$ said; (2) The wind storage system in a year set up cost and operation cost, expressed in $C_{W S} ;$ (3) A year on the distribution network the cost of electricity purchasing $C_{B}$. (4) The wind environment of offset by the energy storage system the cost of $C_{E}$. The expected value model, build a distribution network planning and ensure the node power, node voltage, line transmission power and the wind storage system output fluctuations coefficient is achieved, such as on this basis, the assembly planning society this year's expected value control to a minimum.

Obj.:

$$
\begin{aligned}
& \min Z=C_{L}+C_{W S}+C_{B}-C_{E} \\
& \left\{\begin{array}{l}
C_{L}=\sum_{i=1}^{n_{N}} \sum_{j=1}^{n_{M}} k_{l i j} C_{l i j} x_{l i j}+8760 \sum_{i=1}^{n_{N}} \sum_{j=1}^{n_{M}} \frac{\Delta V_{i j}^{2}}{\left|Z_{i j}\right|} \lambda_{l} C_{D} \\
C_{W S}=\sum_{i=1}^{n_{W S}}\left(k_{w i} C_{w f i} P_{w i}^{\text {rated }}+k_{s i} C_{s f i} S_{i}^{\text {rated }}\right) x_{w s i}+\sum_{i=1}^{n_{W S}}\left(\rho_{w i} C_{w f i} P_{w i}^{\text {rated }}+\rho_{s i} C_{s f i} S_{i}^{\text {rated }}\right) x_{w s i} \\
C_{B}=8760 C_{b} \cdot\left[\sum_{i=1}^{n_{M}}\left(P_{i}^{\prime}-P_{i}\right)-\sum_{i=1}^{n_{W S}} P_{w i} x_{w s i}\right] \\
C_{E}=8760 C_{e} \cdot \sum_{i=1}^{n_{M S}} P_{w s i} x_{w s i}
\end{array}\right.
\end{aligned}
$$

s.t. 


$$
\left\{\begin{array}{l}
\lambda_{l}\left[\sum_{i=1}^{n_{N}}\left(S_{i j}-\frac{\Delta V_{i j}^{2}}{\left|Z_{i j}\right|}\right)-\sum_{i=1}^{n_{N}} S_{j i}\right]+P_{w s j}-P_{j}^{\prime}=0, \forall j \in n_{M} \\
P_{i j} \leq P_{i j}^{\max } \\
0 \leq \Delta V_{i j} \leq \Delta V^{\max } \\
\eta_{i}=1-\frac{P_{w s i}}{P_{w i}} \leq \eta_{0}, \forall i \in n_{W S}
\end{array}\right.
$$

Where: $\mathrm{Z}$ is the planning of the social total cost; $n_{N}$ is distribution network node the number; $n_{M}$ is load node number; $n_{W S}$ is the number feasible access nodes wind storage system; $\mathrm{k}_{1}$ is the entire line fixed investing congress coefficient of average cost; $k_{w}$ and $k_{s}$ are respectively fixed investing congress flat of the whole wind farms as well as the energy storage system; $C_{l i j}$ is line $i j$ fixed investment cost; $C_{w f i}$ and $C_{s f i}$ are respectively wind power and energy storage devices of fixed investment cost at node $i$; $x_{l i j}$ is a new line between $i$ and $j ; x_{w s i}$ is binary decision variables whether there is any access wind storage systems of node $i ; \Delta V_{i j}$ is line voltage of the line $i j ; Z_{i j}$ is impedance of line $i j ; \lambda_{l}$ is distribution network power factor; $P_{w i}$ is planning output power of storage at node $i$; $C_{b}$ is annual power purchase price; and are respectively rated capacity of wind power and storage device at node $i ; \rho_{w i}$ and $\rho_{s i}$ are respectively annual operation rate of wind power and energy storage devices at node $i ; P$ and $P^{\prime}{ }_{i}$ are respectively initial load values and planning level years load values at node $i ; P_{w s i}$ is output expectations of wind storage system at node $i ; C_{e}$ is environmental cost decreased by wind storage; $P_{i j}$ is active power of line $i j ; P_{i j}^{\max }$ is transmission capacity limit of line $i j ; \Delta V^{\max }$ maximum voltage of branch; $\eta_{i}$ is fluctuation coefficient of wind storage system output power at node $i$; $\eta_{0}$ is fluctuation coefficient upper limit of wind storage system output power according specific planning requirements.

Objective function involved in $x_{l}, x_{w s}, P_{\mathrm{w}}^{\text {rated }}, S^{\text {rated }}$ and $P_{w s}$ in certain Extent, affects the social total cost planning. According to the Eq. $1, P_{w}$ and $H$ depends on $S^{\text {rated }}$ value, when natural condition parameters have been determined When $P_{\mathrm{w}}^{\text {rated }}$ decision $P_{w}$, therefore, $P_{\mathrm{w}}^{\text {rated }}$ and $H$ decide to $S^{\text {rated }}$. In expectations $P_{w s}$ storage system in wind power, for the extraction of the history of the wind speed data as the basis, to be able to with Prated $W$. And the corresponding $S^{\text {rated }}$ different combinations wind storage system, to simulate the power output of situation and draw in the output expectations. According to the $P_{\mathrm{w}}^{\text {rated }}$. And $H$ decided $S^{\text {rated }}$ conclusion, shows that $P_{\mathrm{w}}^{\text {rated }}$ and $H$ determines the $P_{w s}$, accordingly can draw, $P_{\mathrm{w}}^{\text {rated }}$ and $H$ decision about beam in the value of $\eta$.

In addition, in the $H$ value is not in all weather conditions wind storage system under the condition of output value of $P_{w}$, and connection to the distribution network causes changes in trend, therefore, only the output fluctuation of wind storage system the scope of the emerging trend of appropriately control can ensure that change the beginning under effective control. Eq. 2 model set a wind system output power fluctuation coefficient of binding conditions, determine the fluctuation coefficient of concrete road is: first, calculate the planned value of the output power and the actual value every year the difference between, then calculate the difference of year-round power output ratio, namely the first wind storage system planned output power $P_{w}$ and wind system the difference between the system output expectations in $P_{w s}$, results divided by $P_{w}$.

All in all, Eq. 2 represents my such as decision-making variables in the model bottom: $x_{l}, x_{w s}$, and $H$ decision variables. To meet all the constraint conditions, can be value of each of the nodes in the distribution network combination, and then compare the cost, you can find the optimal rules scheme. 


\section{The Wind-Light-Storage Mode of Distribution Network Planning}

In this paper, we discuss the wind - light - storage system is mainly refers to through wind power, photovoltaic power generation, and the combination of energy storage system, to ensure that the output power of implementation plan. By selecting the science of light - storage capacity, can make all the output power of the whole system can satisfy the stability constraint conditions, and the cost can be effectively controlled. It has the body of the computation steps as follows:

(1) Using Monte Carlo method to get wind of annual output expectations, and its output power setting for scenery storage system plan.

(2) According to the wind farm output expectations, determine the corresponding with the wind speed value where $v_{0}$, known fan rated wind speed is greater than this value.

(3) Where $v_{0}$ start wind speed value is selected as wind speed, the wind speed is beyond where $v_{0}$ when to where $v_{0}$ power output value of scenery storage system, in the energy storage device storing excess energy from the wind farms and photovoltaic array all the electrical energy; under the condition of the wind speed is lower than where $v_{0}$, scenery storage system system output is based on and where $v_{0}$ the corresponding power value, photovoltaic power generation not enough parts, if still can't make up, should be fill by the energy storage device charge; if photovoltaic remaining after complement, needs to be stored in a reservoir to equipment.

(4) For different wind, light, storage capacity value was simulated, and luck with the model of planning, can be calculated optimal allocation proportion.

\section{Conclusion}

This paper introduces the wind power distribution network access to the two models, are given a complete mathematical model, according to the history of the local meteorological data and negative load growth and can identify various mode of distribution network line additional, the location of the wind power system and the combination of the constant volume.

\section{References}

[1] Wang lei, Gu Jie. Medium voltage distribution network optimal planning of improved par-theno genetic algorithm [J]. Electric power system Journal of system and its automation, 2006 (3) : 72-76.

[2] El-Khattam W, Bhattacharya K, Hegazy Y, et al. Optimal investment planning for distributed generation in acompetitive electricity market .IEEE Trans on Power Sys-tems,2004,19(3):1674-1684.

[3] Cheng miao miao, longyun kang, da-ming xu, et al. Scenery composite power generation capacity of the energy storage unit in the system optimization design [J]. Electric applications, 2006 (6).

[4] Madawala U K, Thrimawithana D J,Nihal K. An ICPT-supercapacitorhybrid system for surge -free power transfer [J] .IEEETrans on Industrial Electronics, 2007,54(6):3287-3297. 